\title{
Faraday Instability in Small Vessels under Vertical Vibration
}

\author{
A Zubiaga1*, D Brunner', F Sager², M Clemens ${ }^{2}$, \\ E Koepf $^{3},{ }^{1}$ G Boiger ${ }^{1}$ \\ 1. Inst. Computational Physics, ZHAW, Winterthur, \\ Switzerland \\ 2. Late Stage Pharmaceutical \& Processing Development, \\ Pharmaceutical Development \& Supplies, PTD Biologics \\ Europe (PTDE-P). F. Hoffmann-La Roche Ltd. Basel, \\ Switzerland. \\ 3. Commercial Product Support, Innovation and \\ Investments, Pharmaceutical Development \& Supplies, PTD \\ Biologics Europe (PTDE-P). F. Hoffmann-La Roche Ltd. \\ Basel, Switzerland.
}

\begin{abstract}
The formation of Faraday waves in a liquid inside a cylindrical vessel under the influence of vertical vibration is studied. The stability thresholds and its mode decomposition are obtained using a linear stability analysis. The stability model is validated with a vibration experiment in a vertical vibration table. The Faraday instability threshold is found for accelerations ranging from 0.1 to 1.0 times the gravitational acceleration. The confinement effect by the vessel introduces cut-off the low frequency modes and the allowed frequencies are discretized. The resulting acceleration stability threshold is high at low frequencies and it is the lowest at medium frequencies, $10-70 \mathrm{~Hz}$, where the discretization of the mode $k$-momenta introduces low stability regions delimited by more stable frequency ranges. The relevance of these characteristics for the agitation of liquids will be discussed.
\end{abstract}

\section{INTRODUCTION}

Understanding the sloshing of liquids inside vessels and preventing its negative effects is a problem that has occupied engineers for a long time. Civil engineers and seismologists have been interested in the effect that earthquakes could have in dams, water reservoirs or oil tanks. The stabilization of the motion of fuel inside tanks has been a major design problem for jet planes and space rockets [1,2]. Another sort of problems arise from the agitation of biological or pharmaceutical liquid solutions inside vessels. Biomolecules have a high propensity to undergo physical instability reactions that are encountered by changes in the threedimensional structure of the active ingredient. In particular, mechanical stress conditions, such as agitation during shipping, can result in denaturation and aggregation, thereby affecting the stability of the products profoundly [3-5]. The transportation degradation is linked to the shear stress induced by the agitated fluid. One important aspect to understand the agitation of aqueous solutions is the stability threshold above which the free surface shows normal modes.

*Corresponding Author: asier.zubiaga@zhaw.ch 
These modes are stationary and can grow large in intensity. Therefore, they can induce a high shear stress on the dissolved product or induce disastrous failure in large dams or fuel tanks.

Exposed to vertical vibrations liquids are prone to show Faraday instabilities when the acceleration grows above certain threshold value [6]. The vessel shape, it's dimensions and the fluid characteristics (mass density, viscosity and surface tension) influence the formation of instabilities [7,8]. The parametric instability under vertical vibration has been extensively studied for inviscid and viscous fluids. Benjamin \& Ursell [9] found that the free surface instability of low viscosity fluid is well approximated by the Mathieu equation for an ideal fluid. However, this model could not account for the viscosity effects. The extension of the model for viscous fluids was done by Kumar \& Tuckerman [10] using a Floquet analysis [11]. By including the bulk viscosity effects in the boundary conditions at the fluids interface, a stability condition for a given surface wave type is obtained. The viscosity effects near the vessel walls and the interface will also have a noticeably effect in the stability threshold $[9,12]$. The main characteristics of the stability threshold, though, remain largely unchanged $[7,13]$.

In this work, the instability threshold for a liquid inside a vessel subjected to vertical vibrations will be studied both theoretically and experimentally. First, a Floquet analysis of the two fluids (water and air) will be performed. The Faraday instability threshold has then been measured experimentally in a vibration table and the experimental results are compared to the theoretical predictions. The viscosity effects caused by the walls, as well as the free surface will be considered during the experimental validation of the model. The main consequences for transportation of the confinement effect in the vessel will be discussed.

\section{EXPERIMENTAL METHODS}

A vial of $6 \mathrm{ml}(r=10 \mathrm{~mm}, h=23 \mathrm{~mm})$ has been filled with $10 \mathrm{~mm}$ of water. The mass density and viscosity are shown in Table 1 .

Table 1: Mass density and dynamic viscosity of water and air.

\begin{tabular}{lcc}
\hline & Mass density $\left(\mathbf{K g} / \mathbf{m}^{3}\right)$ & Dynamic viscosity $(\mathbf{m P a} \cdot \boldsymbol{s})$ \\
\cline { 2 - 3 } Water & 1000 & 1 \\
Air & 1.146 & 0.0172 \\
\hline
\end{tabular}

The vibration experiments were conducted in a Lasmont Field-to-Lab ${ }^{\circledR}$ table sized 1000 Vibration Testing System (vibration table from now on) which performs sinusoidal and random vibration tests in the $1-300 \mathrm{~Hz}$ frequency range and maximum peak-to-peak amplitude of $10.2 \mathrm{~cm}$. A hydraulic actuator and a hydraulic power supply drive the vibration system. The parametric excitation has been performed at constant acceleration $(0.1 G, 0.18 G$, $0.32 G, 0.56 G$ and $1.0 G$, where $G=9.81 \mathrm{~m} / \mathrm{s}^{2}$ ) and a frequency scan has been done from $5 \mathrm{~Hz}$ to $120 \mathrm{~Hz}$ with 2 octaves. The vial was carefully mounted on top of a white polyoxymethylene (POM) base. The camera was at rest in the laboratory and it recorded the vial laterally. The framing was chosen so that the vial and the white base were framed along the whole experiment. The POM base was used as reference point for aligning the position of the vertically displaced vial in all the frames during post processing.

The free surface of the vial has been recorded with a Mikrotron MotionBlitz EoSensR high speed camera equipped with a Complementary metal-oxide-semiconductor (CMOS) sensor 
that can record up to a maximum of 506 frames per second ( $f p s)$ at $1280 \times 1024$ pixel and a 8-bit monochrome resolution. The shutter aperture time of $\sim 1 \mathrm{~ms}$ is enough to take sharp images even at the highest frequency chosen. The vibration test has been recorded at $25 \mathrm{fps}$ in a single movie. This recording rate is does not allow following the evolution of the waves at high frequencies, but the wave intensity could be still measured as long as the wave peakto-peak amplitude is larger than the width of the fluid meniscus (see Figure 1d). At high frequencies, the vibration amplitude $a_{0}=A_{0} / \omega^{2}$ decreases strongly. Consequently, no waves larger than the meniscus could be measured (see Figure 1e).

The movie has been imported and processed frame by frame in MATLAB®. First, the vertical vibration movement has been subtracted by aligning the position of the POM base in all the frames. Then, a reference frame $\left(F r_{0}\right)$ has been defined where the fluid is at complete rest and it has been compared to the frames $\left(F r_{i}\right)$ with a moving vial. The $\mathrm{N}$ pixel average difference between $F r_{i}$ and $F r_{0} D(i)=\left[\sum_{i=1}^{N}\left(F r_{i}-F r_{0}\right)^{2} / N\right]^{0.5}$ has been used as an indicator for surface movement (wave intensity from now on). To reduce noise, the processed region has been restricted to a rectangular region around the water-air interface (see Figure 1 for more details). Finally, a running average with 25 frames of width was applied to minimize the noise from imperfect alignment of the frames and other effects such as such as light reflections.
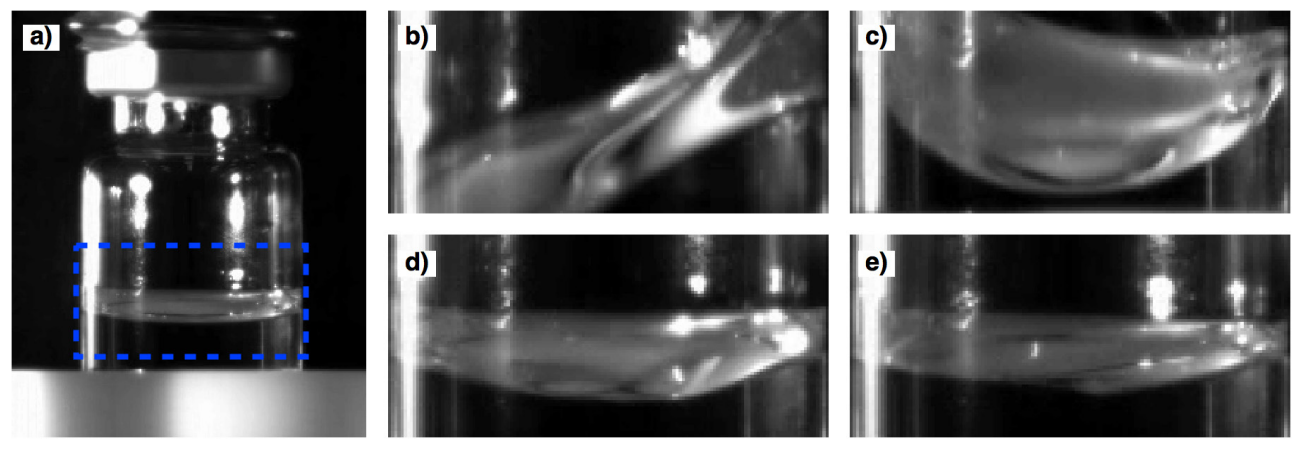

Figure 1. - Frames used for the analysis of the wave intensity. Panel a) shows a frame with the vial at rest and a flat water-air interface. The wave intensity has been calculated from the framed region. Panels b) - e) show the framed region for an acceleration of $1.0 G$ and different vibration frequency. Panel b) and c) show the water-air interface with waves. Panel d) shows the water-air interface at high frequencies, when the wave intensity becomes small. Finally, panel e) shows the flat water-air interface at very high frequencies.

\section{STABILITY ANALYSIS}

The stability of the free surface of a fluid in contact with air and inside a closed vessel are analyzed. The vessel has cylindrical shape and its dimensions are comparable to the vial used in the experiments $(r=10 \mathrm{~mm}$, water height of $10 \mathrm{~mm}$ and air column height of $6 \mathrm{~mm}$ ). At rest, the fluids are distributed in their gravitationally stable configuration, with the air located on top of the water. The dynamics of both fluids are characterized by their mass density $\rho$ and viscosity $\eta$. The dissipation due to viscosity effects at the vessel walls and the interface has a significant influence but it does not change the main characteristics of the instability 
[14], therefore only bulk viscosity effects have been included. The dynamics of the free surface is governed by the surface tension $\sigma=0.072 \mathrm{~N} / \mathrm{m}$.

The vertical vibration are studied in the reference frame of the vessel. The acceleration is set along the vertical direction with a constant gravitational term and an oscillating term

$$
A(t)=-G+A_{0} \cos (\omega t)
$$

$A_{0}$ is the acceleration amplitude and $\omega=2 \pi f$. The fluids are described using the NavierStokes equations for incompressible fluids

$$
\begin{aligned}
{\left[\partial_{t}+\left(\vec{U}_{i} \cdot \vec{\nabla}\right)\right] \vec{U}_{i} } & =\frac{-1}{\rho_{i}} \vec{\nabla} P_{i}+\frac{\eta_{i}}{\rho_{i}} \nabla^{2} \vec{U}_{i}+A(t) \hat{z} \\
& \vec{\nabla} \cdot \vec{U}_{i}=0
\end{aligned}
$$

$\mathrm{P}$ is the pressure, $\hat{z}$ is the unit vector along the vertical direction and the subscript $i=1,2$ correspond to the upper (lighter) and lower (heavier) fluids, respectively. Non-slip boundary conditions are imposed for the velocity at the vessel walls

$$
\begin{gathered}
\vec{U}_{i}=0 \\
{[\vec{n} \cdot \vec{\nabla}]\left[\vec{U}_{i} \cdot \vec{n}\right]=0}
\end{gathered}
$$

Where $\vec{n}$ is a vector normal to the vessel wall. In the interface the normal velocities $U_{\perp}=$ $\vec{U} \cdot \vec{t}$ are continuous

$$
\begin{aligned}
U_{1 \perp} & =U_{2 \perp} \\
\vec{\nabla} U_{1 \perp} \cdot \vec{t} & =\vec{\nabla} U_{2 \perp} \cdot \vec{t}
\end{aligned}
$$

The kinematic free surface condition is given by [15]

$$
\left[\partial_{t}+\left(\vec{U}_{i} \cdot \vec{\nabla}\right)\right] \xi=U_{i, z}
$$

Where $\xi$ is the position of the interface. Finally, when the surface curvature is small the jump in the normal component of the stress tensor across the interface $\pi_{i, r s}=-P_{i} \delta_{r s}+$ $\eta_{i}\left(\partial_{r} \vec{U}_{i, s}+\partial_{s} \vec{U}_{i, r}\right)$ is

$$
\left(\pi_{1, z z}-\pi_{2, z z}\right)=-\sigma \nabla_{H}^{2} \xi
$$

Where the subscript $\mathrm{H}$ refers to the horizontal projection along $\{\mathrm{x}, \mathrm{y}\}$ directions. In the initial state, the free surface is perfectly horizontal. This will define the $\mathrm{z}=0$ reference position for the vertical axis and the rest state $\vec{U}_{i}^{0}=0, P_{n}^{0}(t)=\rho_{n} A(t) z$. For small velocities and deviations from the flat surface, the Navier-Stokes equations can be linearized 


$$
\begin{gathered}
\vec{U}_{i}=\vec{u}_{i} \\
P_{i}=P_{i}^{0}+p_{i} \\
\partial_{t} \vec{u}_{i}=\frac{-1}{\rho_{i}} \vec{\nabla} p_{i}+\frac{\eta_{i}}{\rho_{i}} \nabla^{2} \vec{u}_{i} \\
\vec{\nabla} \cdot \vec{U}_{i}=\vec{\nabla}_{H} \cdot \vec{u}_{i, H}+\partial_{z} u_{i, z}=0
\end{gathered}
$$

For the following analysis, the position of the free surface can be assumed to be located at $z=0$. The vibrational acceleration does not appear now in the equations and using the properties of the differential operators and focusing in the vertical velocity, the Navier-Stokes can be written as

$$
\left(\partial_{t}-\frac{\eta_{i}}{\rho_{i}} \nabla^{2}\right) \nabla^{2} u_{i, z}=0
$$

The boundary conditions at the upper $\left(z=h_{1}\right)$ and lower $\left(z=-h_{2}\right)$ walls and the free surface are

$$
\begin{gathered}
u_{1, z}\left(h_{1}\right)=\partial_{z} u_{1, z}\left(h_{1}\right)=0 \\
u_{2, z}\left(-h_{2}\right)=\partial_{z} u_{2, z}\left(-h_{2}\right)=0 \\
u_{1, z}(0)=u_{2, z}(0) ; \partial_{z} u_{1, z}(0)=\partial_{z} u_{2, z}(0) \\
\partial_{t} \xi=\left.u_{z}\right|_{z=0} \\
\delta\left(\rho \partial_{t}-\eta \nabla^{2}\right) \partial_{z} u_{z}-2 \delta \eta \nabla_{H}^{2} \partial_{z} u_{z}=\left(\sigma \nabla_{H}^{4}-\delta \rho A(t) \nabla_{H}^{2}\right) \xi
\end{gathered}
$$

The jumps at the interface are represented by $\delta \rho=\left(\rho_{1}-\rho_{2}\right)$ for the fluid density, and $\delta \eta=\left(\eta_{1}-\eta_{2}\right)$ for the fluid viscosity. Equation (9) together with the non-slip boundary conditions at the vertical wall defines the solution in axial coordinates $(\mathrm{r}, \theta, \mathrm{z})$ as

$$
u_{j, z}=J_{m}\left(\lambda_{m n} r\right) \sin (m \theta) w_{j, m n}(z, t)
$$

$J_{m}\left(\lambda_{m n} r\right)$ is the Bessel function of the first kind for mode $(m, n)$ and $1 / \lambda_{m n}$ is the position of its nth root. The Faraday waves are composed by $(m, n)$ modes, each with a distinct parametric excitation profile. The allowed modes are discretized and bounded from below by $\lambda_{11}=2.40483 / r$. For an $(m, n)$ mode equation $(8)$ is rewritten as

$$
\left[\partial_{t}-\frac{\eta_{j}}{\rho_{j}}\left(\partial_{z z}-\lambda_{m n}^{2}\right)\right]\left(\partial_{z z}-\lambda_{m n}^{2}\right) w_{j, m n}=0
$$

The solutions are found expanding $w_{j, m n}$ in a Fourier series

$$
w_{j, m n}(z, t)=e^{(\mu+i \alpha) t} \sum_{k=-\infty}^{\infty} w_{j, m n}^{k}(z) e^{i k \omega t}
$$

The exponent $\mu+i \alpha$ is the Floquet exponent and $i=\sqrt{-1}$ the imaginary number. Only the harmonic $(\alpha=0)$ and subharmonic $(\alpha=1 / 2)$ cases lead to stationary solutions. The eigenfunctions $w_{j n}$ of Equation (11) can be found now from 


$$
\left[\mu+i(\alpha+k \omega)-v_{j}\left(\partial_{z z}-\lambda_{m n}^{2}\right)\right]\left(\partial_{z z}-\lambda_{m n}^{2}\right) w_{j, m n}^{k}=0
$$

where $v_{j}=\eta_{j} / \rho_{j}$ is the kinematic viscosity. The solutions are written as a function of $q_{j, m n}^{k}=$ $\sqrt{\lambda_{m n}^{2}+\frac{\mu+i(\alpha+k \omega)}{v_{j}}}$. Leaving the liquid and the mode indexes implicit the functions $w^{k}(z)$ is

$$
w^{k}(z)= \begin{cases}a^{k} e^{\lambda z}+b^{k} e^{-\lambda z}+c^{k} e^{q^{k} z}+d^{k} e^{-q^{k} z} & q^{k}>0 \\ a^{k} e^{\lambda z}+b^{k} e^{-\lambda z}+c^{k} \lambda e^{\lambda z}+d^{k} \lambda e^{-\lambda z} & q^{k}=0\end{cases}
$$

The kinematic condition implies that the interface position is

$$
\begin{gathered}
\xi(r, \theta, t)=e^{(\mu+i \alpha) t} \sum_{k=-\infty}^{\infty} \xi^{k} e^{i k \omega t} \\
\xi^{k}[\mu+i(\alpha+k \omega)]=\left.u_{j, z}\right|_{z=0}
\end{gathered}
$$

and can be used to write the velocity coefficients $a^{k}, b^{k}, c^{k}$ and $d^{k}$ as a function of $\xi$. The vibrational term of the pressure jump condition

$$
\delta \rho \lambda_{m n}^{2} \frac{A_{0}}{2}\left(e^{i \omega t}+e^{-i \omega t}\right)
$$

couples the terms of the Fourier series, yielding a generalized eigenvalue problem

$$
\hat{A} \xi=A_{0} \hat{B} \xi
$$

Where $\hat{A}$ is a diagonal complex matrix and $\hat{B}$ a banded matrix. The lowest real eigenvalue $A_{0}$ for $\mu=0$ gives the marginal stability boundary of the harmonic $\alpha=0$ and subharmonic $\alpha=1 / 2$ branches.

\section{RESULTS \& DISCUSSION}

\subsection{Simulation results}

The generalized eigenvalue problem has been solved numerically for each cylindrical mode $(m, n)$ and excitation frequency $\omega$. In a first step, the eigenfunction coefficients are obtained from the boundary and interface conditions by solving a non-homogeneous linear system. Then, the matrices $\hat{A}$ and $\hat{B}$ from equation (17) can be obtained from the jump in the shear stress at the free surface. The eigenvalue problem is solved numerically and the lowest real eigenvalue $A_{0}$ is the excitation threshold for the mode at a given frequency [14].

Figure 2 shows the stability threshold for the lowest modes of the cylindrical vial. Each mode has a distinct minimum acceleration, the critical acceleration, where it can be resonantly excited. In general, the subharmonic branch has a lower critical acceleration and occurs at larger vibration frequencies. The overall stability threshold for a given frequency is the lowest acceleration threshold among the subharmonic or harmonic branches of all the $(m, n)$ modes. In general, a different mode is excited depending on the frequency and the acceleration threshold is rather complex due to the non-trivial dependence on the excitation frequency. Between $15.0 \mathrm{~Hz}$ and $16.2 \mathrm{~Hz}$, the subharmonic $(1,2)$ mode is onset. At slightly lower 
frequencies mainly the subharmonic $(1,1)$ mode is onset. However, in narrow frequency ranges at $21.1 \mathrm{~Hz}$ and at $25.2 \mathrm{~Hz}$, the subharmonic $(2,1)$ and the subharmonic $(1,4)$ modes are excited.

Due to the lower bound on the k-momentum of the modes, the acceleration threshold at low frequencies $(f<10 \mathrm{~Hz})$ is large. The absolute critical acceleration corresponds to the $(1,1)$ subharmonic mode at $\sim 17 \mathrm{~Hz}$ with $a_{c}=0.15 \mathrm{~m} / \mathrm{s}^{2}$. Higher modes have larger critical accelerations that grow sharply with the frequency as the creation of waves at high frequencies in water becomes increasingly difficult.

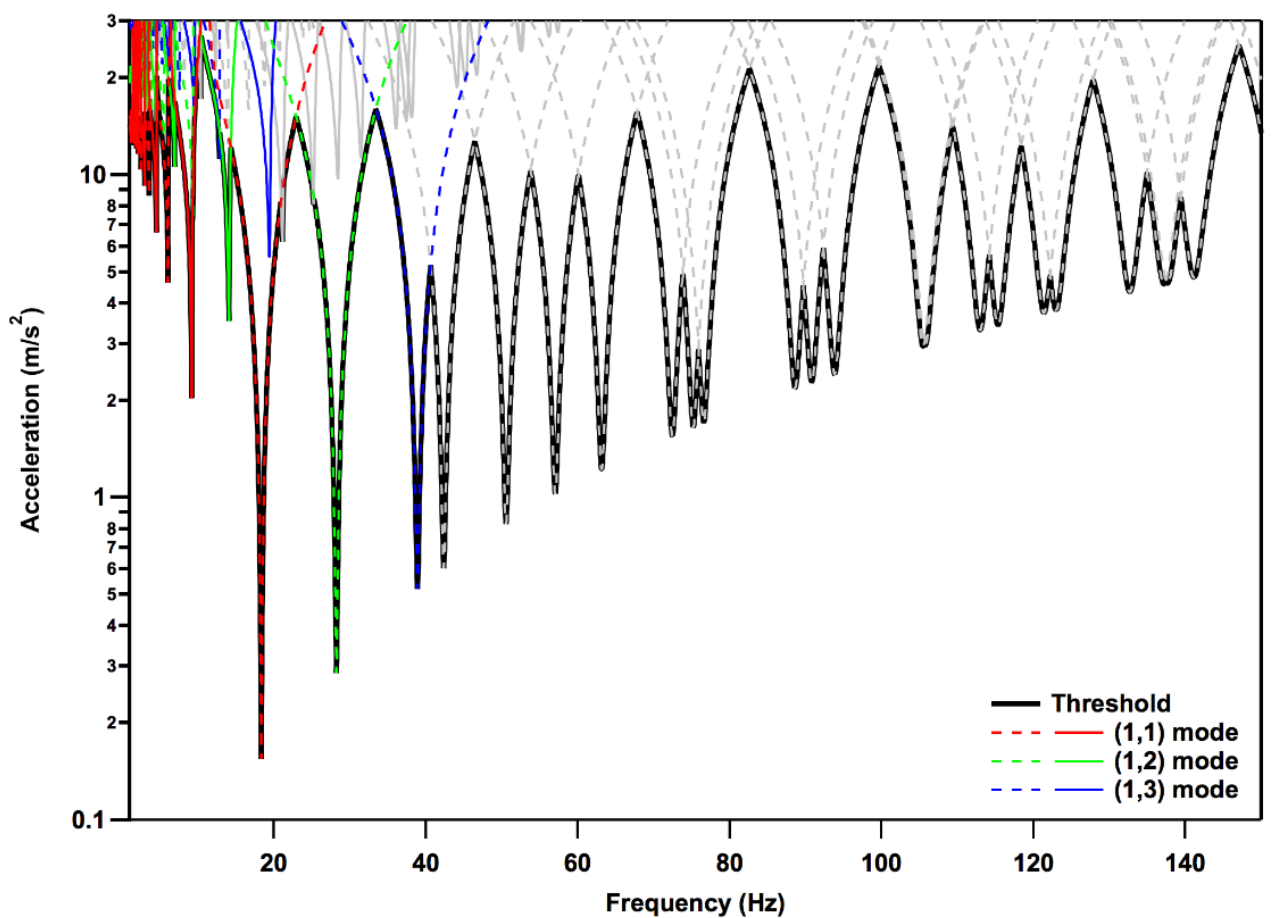

Figure 2. - Calculated stability threshold and mode composition. The subharmonic modes are shown in broken lines and the harmonic modes in full lines. The lowest 3 modes are highlighted in color.

\subsection{Experimental results}

The vertical vibration experiments of the vial in a vibration table represent a validation of the stability analysis. The five chosen accelerations, $0.1 G, 0.18 G, 0.32 G, 0.56 G$ and $1.0 G$, cover a wide range of the dynamics of the Faraday waves. At the lowest acceleration, shallow waves are created at narrow frequency ranges. As the acceleration is increased from $0.18 G$ to $0.56 G$, the frequency ranges where waves are created widen and also the intensity of the waves increases. At the higher acceleration, waves are created in the full frequency range.

The wave intensity is plotted in Figure 3 versus the corresponding vibration frequency. At $0.1 G$, waves were observed only at a narrow frequency interval at $\sim 13 \mathrm{~Hz}$. At an acceleration of $0.18 \mathrm{G}$, the intensity of the peak increases and a new one appears at $\sim 26 \mathrm{~Hz}$. Two weak 
bands appear at $\sim 42 \mathrm{~Hz}$ and $\sim 58 \mathrm{~Hz}$ at $0.32 \mathrm{G}$ and they become stronger at $0.56 \mathrm{G}$. Finally, at $1.0 G$ waves are observed in the whole frequency range and the wave intensity has large variations that can be abrupt at certain frequency values.

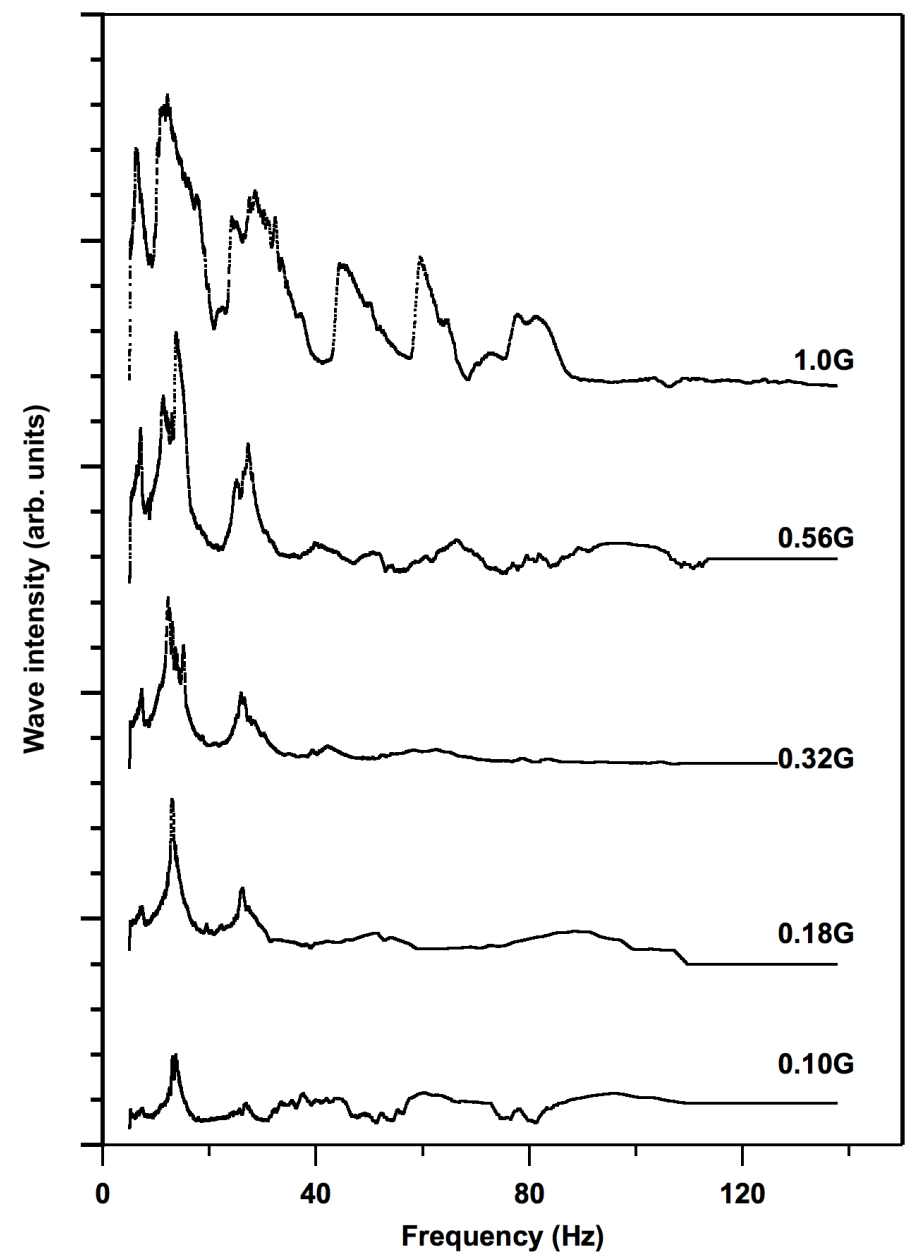

Figure 3. - Wave intensity change with excitation frequency for the five measured accelerations. The curves have been stacked vertically for clarity.

In figure 4, the frequency dependence of the wave intensity is compared to the calculated stability threshold in an acceleration vs frequency plot. The absolute critical acceleration is at a frequency, $13 \mathrm{~Hz}$, lower than predicted by the linear stability model, $17 \mathrm{~Hz}$. This discrepancy is resulting from the viscosity effects near the walls and the free surface. Between 10 and $70 \mathrm{~Hz}$, the acceleration threshold is low close to the resonance frequencies of the modes. The resonance frequencies form tongues separated by higher stability frequency ranges as a consequence of the discretization of the allowed mode $k$-momenta. Although the measurements do not thoroughly allow to measure the steep increase of the stability threshold at high frequencies, the strong decrease of the wave intensity at the highest frequencies and at all accelerations is clearly compatible with this feature. 

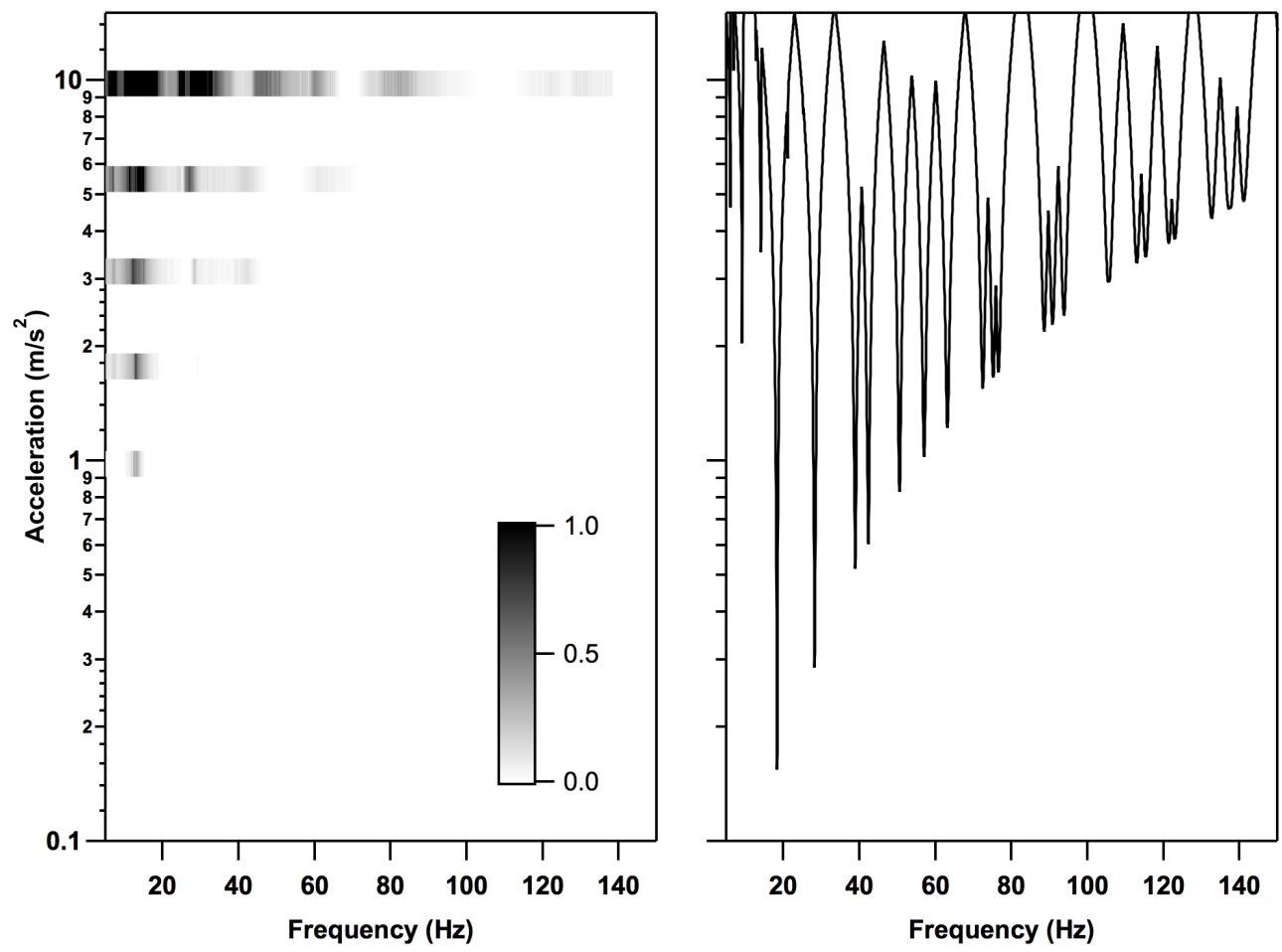

Figure 4. - The acceleration behavior plotted versus frequency in the experiments (left panel) and in the stability analysis (right panel). In the left panel, the intensity of the color represents the wave intensity in arbitrary units.

Finally, the fast changes of the wave intensity at the highest acceleration value come from transition thresholds where the mode composition of the Faraday instability changes abruptly. The creation (or the disappearance) of mode components originate from (non-linear) transitions above the instability onset threshold [16,17]. The stability analysis of Figure 2 shows a linear approximation to the mode decomposition of the instability above the stability threshold.

The liquid viscosity and surface tension, as well as the vessel size and filling level, have noticeably effects on the stability threshold and the transportation stress. Horizontal vibrations should also be present in real transport conditions and they will introduce waves already at low excitation accelerations. Therefore, agitation stress can already increase substantially below the stability threshold, when normal modes have not yet been excited. In more realistic situations, non-linear effects coming from multi-frequency simultaneous excitation [18] or mode interaction [17] can alter the value and frequency dependence of the stability threshold. These factors need to be considered in order to assess the agitation stress. 


\section{CONCLUSIONS}

A full study of the stability threshold of a liquid in a small vessel under vertical vibrations has been performed. The mode components of the Faraday instability have been calculated with a linear stability theory in a wide frequency range, ranging from $5 \mathrm{~Hz}$ to $150 \mathrm{~Hz}$. The theory has been validated by measuring water in small vials under vertical vibrations. A qualitative agreement with the theory is observed, and the disagreement in the position of the stability tongues can be well explained by the non-linear viscosity effects exerted by the container walls. The existence of a low stability region between 10 and $70 \mathrm{~Hz}$ has been confirmed by the experiments. The constrain in the allowed k-momenta, enforced by the vial dimensions, creates low stability tongues. On the one hand, the stability theory predicts a lower kmomentum cut-off in the stability threshold, which has a protective effect on the liquid against low frequency instabilities. On the other hand, at frequencies larger than $20 \mathrm{~Hz}$, the instability threshold increases steeply due to the intrinsic response of the liquid to high excitation frequency. The existence at medium frequencies of low stability threshold tongues makes highly non-trivial the study of agitation effects.

\section{REFERENCES}

[1] Abramson HN. Dynamic behavior of liquids in moving containers. Appl Mech Rev. 1963;16(7):501-6.

[2] Cooper RM. Dynamics of liquids in moving containers. ARS J. 1960;30:725-9.

[3] Torisu T, Maruno T, Hamaji Y, Ohkubo T, Uchiyama S. Synergistic Effect of Cavitation and Agitation on Protein Aggregation. J Pharm Sci. 2017 Feb 1;106(2):5219.

[4] Bai G, Bee JS, Biddlecombe JG, Chen Q, Leach WT. Computational fluid dynamics (CFD) insights into agitation stress methods in biopharmaceutical development. Int J Pharm. 2012 Feb 28;423(2):264-80.

[5] Fleischman ML, Chung J, Paul EP, Lewus RA. Shipping-Induced Aggregation in Therapeutic Antibodies: Utilization of a Scale-Down Model to Assess Degradation in Monoclonal Antibodies. J Pharm Sci. 2017 Apr 1;106(4):994-1000.

[6] Faraday M. XVII. On a peculiar class of acoustical figures; and on certain forms assumed by groups of particles upon vibrating elastic surfaces. Philos Trans R Soc Lond. 1831 Jan 1;121:299-340.

[7] Henderson DM, Miles JW. Single-mode Faraday waves in small cylinders. J Fluid Mech. 1990 Apr;213:95-109.

[8] Bechhoefer J, Ego V, Manneville S, Johnson B. An experimental study of the onset of parametrically pumped surface waves in viscous fluids. J Fluid Mech. 1995 Apr;288:325-50.

[9] Benjamin TB, Ursell FJ. The stability of the plane free surface of a liquid in vertical periodic motion. Proc R Soc Lond A. 1954 Sep 22;225(1163):505-15.

[10] Kumar K, Tuckerman LS. Parametric instability of the interface between two fluids. J Fluid Mech. 1994 Nov;279:49-68.

[11] Floquet G. Sur les équations différentielles linéaires à coefficients périodiques. Ann Sci LÉcole Norm Supér. 1883;12:47-88.

[12] Miles JW. Surface-wave damping in closed basins. Proc R Soc Lond A. 1967 Mar 21;297(1451):459-75. 
[13] Dodge FT, Kana D, Abramson HN. Liquid surface oscillations in longitudinally excited rigid cylindrical containers. AIAA J. 1965 Apr 1;3(4):685-95.

[14] Batson W, Zoueshtiagh F, Narayanan R. The Faraday threshold in small cylinders and the sidewall non-ideality. J Fluid Mech. 2013 Aug;729:496-523.

[15] Lamb H. Hydrodynamics. 6th edition. Cambridge University Press; 1932.

[16] Ciliberto S, Gollub JP. Chaotic mode competition in parametrically forced surface waves. J Fluid Mech. 1985 Sep;158:381-98.

[17] Tipton CR, Mullin T. An experimental study of Faraday waves formed on the interface between two immiscible liquids. Phys Fluids. 2004 May 25;16(7):2336-41.

[18] Batson W, Zoueshtiagh F, Narayanan R. Two-frequency excitation of single-mode Faraday waves. J Fluid Mech. 2015 Feb;764:538-71. 
